\title{
Alfven wave interactions within the Hall-MHD description
}

\author{
G Brodin and Lennart Stenflo
}

\section{Linköping University Post Print}

\section{Tweet}

N.B.: When citing this work, cite the original article.

Original Publication:

G Brodin and Lennart Stenflo, Alfven wave interactions within the Hall-MHD description, 2013, Journal of Plasma Physics, (79), 909-911.

http://dx.doi.org/10.1017/S0022377813000640

Copyright: Cambridge University Press (CUP)

http://www.cambridge.org/uk/

Postprint available at: Linköping University Electronic Press

http://urn.kb.se/resolve?urn=urn:nbn:se:liu:diva-99404 


\title{
Alfven wave interactions within the Hall-MHD description*
}

\author{
G. B R O D I N ${ }^{1}$ and L. STE N F L O \\ ${ }^{1}$ Department of Physics, Umeå University, Umeå SE-901 87, Sweden \\ (Gert.Brodin@physics.umu.se) \\ ${ }^{2}$ Department of Physics, Linköping University, Linköping SE-581 83, Sweden
}

(Received 15 April 2013; revised 14 May 2013; accepted 14 May 2013; first published online 28 June 2013)

\begin{abstract}
We show that comparatively simple expressions for the Alfven wave coupling coefficients can be deduced from the well-known Hall-magnetohydrodynamics (MHD) model equations.
\end{abstract}

\section{Introduction}

The nonlinear properties of Alfven waves (e.g. Stasiewicz et al. 2000) are most important ingredients in explaining several kinds of space observations (e.g. Chmyrev et al. 1988; Petviashvili and Pokhotelov 1992; Sundkvist et al. 2005). Consequently, there are numerous papers (e.g. Shukla et al. 1982; Shukla and Stenflo 1995; Voitenko et al. 1998; Fedun et al. 2004; Ruderman and Caillol 2008) devoted to explanations of various kinds of Alfven waves. Very recent papers (e.g. Rudakov et al. 2011; Kumar and Sharma 2011; Zhao et al. 2011, 2012; Das et al. 2012; Kumar 2012) confirm the increasing interest in such phenomena.

Zhao et al. (2011) thus considered the interaction between three Alfven waves, where one wave propagates along the external magnetic field whereas the other two waves have oblique propagation directions. Furthermore, Zhao et al. (2012) showed that such couplings lead to large-scale self-organized convective vortex and vortex chain configurations (e.g. Chmyrev et al. 1988).

In the present paper we are going to point out that the well-known Hall-magnetohydrodynamics (MHD) equations yield results (Brodin and Stenflo 1990) that seem to have been overlooked for more than two decades, but now anyhow ought to be considered.

\section{Equations}

Twenty-five years ago we (Brodin and Stenflo 1988) adopted the well-known ideal MHD equations. Thus, we started with the following equations:

$$
\begin{gathered}
\frac{\partial \rho}{\partial t}+\nabla \cdot(\rho \mathbf{v})=0, \\
\rho \frac{d \mathbf{v}}{d t}=-c_{s}^{2} \nabla \rho+\frac{(\nabla \times \mathbf{B}) \times \mathbf{B}}{\mu_{0}},
\end{gathered}
$$

and

$$
\frac{\partial \mathbf{B}}{\partial t}=\nabla \times(\mathbf{v} \times \mathbf{B})
$$

* In memory of Padma Shukla - a great scientist and a good friend. to consider the interaction between magnetosonic and Alfven waves. Here $d / d t=\partial / \partial t+\mathbf{v} \cdot \nabla, \rho$ is the density, $\mathbf{v}$ is the fluid velocity, $\mathbf{B}$ is the magnetic field, and $c_{s}$ is the ion sound velocity. Studying a uniform magnetized plasma with an external magnetic field $\mathbf{B}=B_{0} \hat{\mathbf{z}}$, we first linearized (1)-(3) to re-derive the following linear dispersion relation:

$$
\left(\omega^{4}-\omega^{2} k^{2}\left(c_{A}^{2}+c_{s}^{2}\right)+k_{z}^{2} k^{2} c_{A}^{2} c_{s}^{2}\right)\left(\omega^{2}-k_{z}^{2} c_{A}^{2}\right)=0,
$$

where $c_{A}=\left(B_{0} / \mu_{0} \rho_{0}\right)^{1 / 2}$ is the Alfvén speed, $\omega$ is the wave frequency, $k$ is the magnitude of the wave vector $\mathbf{k}$, and $k_{z}$ is its $z$-component. We then used the weakly nonlinear version of (1)-(3) to consider the interaction between three magnetosonic waves, two magnetosonic waves and one Alfven wave, one magnetosonic wave and two Alfven waves as well as three Alfven waves. In the last case, it turned out that the coupling coefficients were zero.

In order to reconsider the interaction between three Alfven waves, we then generalized (3) to replace it with the corresponding Hall-MHD equation,

$$
\frac{\partial \mathbf{B}}{\partial t}=\nabla \times\left(\mathbf{v} \times \mathbf{B}-\frac{m_{i}}{e} \frac{d \mathbf{v}}{d t}\right),
$$

where $e$ and $m_{i}$ are the ion charge and mass respectively. The linear dispersion relation then turned out to be (Brodin and Stenflo 1990)

$$
\begin{aligned}
D(\omega, \mathbf{k})= & \left(\omega^{4}-\omega^{2} k^{2}\left(c_{A}^{2}+c_{s}^{2}\right)+k_{z}^{2} k^{2} c_{A}^{2} c_{s}^{2}\right)\left(\omega^{2}-k_{z}^{2} c_{A}^{2}\right) \\
& -\frac{\omega^{2} k_{z}^{2} k^{2}\left(\omega^{2}-k^{2} c_{s}^{2}\right) c_{A}^{4}}{\omega_{c i}^{2}}=0,
\end{aligned}
$$

where $\omega_{c i}=e B_{0} / m_{i}$ is the ion cyclotron frequency. The dispersion relation (6) was later reconsidered (e.g. Hirose et al. 2004) and renamed and discussed further (Bellan 2012).

As the general coupling coefficients for three-wave interaction between Hall-MHD waves derived by Brodin and Stenflo (1990) turned out to be rather complex, the case of three Alfven waves was not further discussed at that time. In order to proceed and simplify the formulas, we shall therefore, in the present paper, only consider the case where $k^{2} c_{A} \sim k^{2} c_{s} \sim k \omega \ll k_{\perp} \omega_{c i}$ for all interacting 
waves. Using (6), the root corresponding to the shear Alfvén wave can then be approximated by

$$
\omega^{2} \simeq \frac{k_{z}^{2} c_{A}^{2}}{1+k^{2} k_{z}^{2} \lambda_{e}^{2} / k_{\perp}^{2}}\left(1+\frac{k^{4} \rho^{2}}{k_{\perp}^{2}}\right),
$$

where $\rho=c_{s} / \omega_{c i}, \lambda_{e}=c_{A} / \omega_{c i}$, and $k_{\perp}$ is the magnitude of the wavenumber perpendicular to $\hat{\mathbf{z}}$. We note that the factor $\left(1+k^{4} \rho^{2} / k_{\perp}^{2}\right)$ in the numerator of (7) agrees with that for kinetic Alfven waves if $k_{\perp}^{2} \gg k_{z}^{2}$. However, the factor $\left(1+k^{2} k_{z}^{2} \lambda_{e}^{2} / k_{\perp}^{2}\right)$ in the denominator does not in general agree with the factor $\left(1+k_{\perp}^{2} \lambda_{e}^{2}\right)$ for the inertial Alfven waves (Brodin et al. 2007), although they are qualitatively similar if $k_{z} \sim k_{\perp}$.

Next we consider the resonant interaction between three Hall-MHD waves which satisfy the matching conditions

$$
\omega_{3}=\omega_{1}+\omega_{2}
$$

and

$$
\mathbf{k}_{3}=\mathbf{k}_{1}+\mathbf{k}_{2} .
$$

One then finds the equations (Brodin and Stenflo 1990)

$$
\left(\frac{\partial}{\partial t}+\mathbf{v}_{g 1,2} \cdot \nabla\right) \rho_{1,2}=-\frac{1}{\partial \tilde{D}_{1,2} / \partial \omega_{1,2}} C \rho_{2,1}^{*} \rho_{3}
$$

and

$$
\left(\frac{\partial}{\partial t}+\mathbf{v}_{g 3} \cdot \nabla\right) \rho_{3}=\frac{1}{\partial \tilde{D}_{3} / \partial \omega_{3}} C \rho_{1} \rho_{2},
$$

where $\rho_{j}(j=1,2,3)$ is the density perturbation of wave $j$ and

$$
\tilde{D}_{j}\left(\omega_{j}, \mathbf{k}_{j}\right)=\frac{\left(\omega_{j}^{2}-k_{j}^{2} c_{s}^{2}\right) D\left(\omega_{j}, \mathbf{k}_{j}\right)}{\left(\omega_{j}^{2}-k_{j z}^{2} c_{A}^{2}\right) \omega_{j}^{2} k_{j \perp}^{2} k_{j}^{2} c_{A}^{2}} .
$$

The general expression for the coupling coefficient $C$ for arbitrary Hall-MHD wave modes governed by (6) is given in Brodin and Stenflo (1990). For the case of three shear Alfvén waves with $k c_{A} \sim k c_{s} \sim \omega \ll \omega_{c i}$, where the approximate dispersion relation (7) applies for all interacting waves, the expression for the coupling coefficient immediately reduces to

$$
\begin{aligned}
C \simeq & \frac{\omega_{1} \omega_{2} \omega_{3}}{\rho_{0} k_{1 \perp}^{2} k_{2 \perp}^{2} k_{3 \perp}^{2}}\left[\frac{\mathbf{K}_{3} \cdot \mathbf{K}_{2}^{*}}{\omega_{1}} k_{1 \perp}^{2}+\frac{\mathbf{K}_{3} \cdot \mathbf{K}_{1}^{*}}{\omega_{2}} k_{2 \perp}^{2}\right. \\
& \left.+\frac{\mathbf{K}_{1}^{*} \cdot \mathbf{K}_{2}^{*}}{\omega_{3}} k_{3 \perp}^{2}-\frac{k_{1 \perp}^{2} k_{2 \perp}^{2} k_{3 \perp}^{2}}{\omega_{1} \omega_{2} \omega_{3}} c_{s}^{2}\right],
\end{aligned}
$$

where

$$
\mathbf{K}_{j} \simeq \mathbf{k}_{j \perp} \frac{\left(\omega_{j}^{2}-k_{j z}^{2} c_{s}^{2}\right)}{\omega_{j}^{2}}+\frac{i \hat{\mathbf{z}} \times \mathbf{k}_{j \perp}\left(\omega_{j}^{2}-k_{j}^{2} c_{s}^{2}\right) k_{j z}^{2} c_{A}^{2}}{\omega_{c i} \omega_{j}\left(\omega_{j}^{2}-k_{j z}^{2} c_{A}^{2}\right)} .
$$

Next we rewrite the three wave equations (10) and (11) in terms of the velocity amplitude, where the velocity can be expressed in terms of the density from $\mathbf{v}_{j}=$ $\rho_{j} \mathbf{K}_{j} \omega_{j} / k_{j \perp}^{2} \rho_{0}$ (Brodin and Stenflo 1990). Simplifying the formulas (12), (13), and (14) using the dispersion relation (7) for each wave, we then find that the coupled equations reduce to

$$
\begin{aligned}
& \left(\frac{\partial}{\partial t}+\mathbf{v}_{g 1,2} \cdot \nabla\right) v_{1,2} \simeq \frac{k_{z 1,2}^{2} c_{A}^{2}}{2 \omega_{1,2} k_{1 \perp} k_{2 \perp} k_{3 \perp} \omega_{c i}}\left[k_{1}^{2} \mathbf{k}_{3 \perp} \cdot \mathbf{k}_{2 \perp}\right. \\
& \left.\quad+k_{2}^{2} \mathbf{k}_{1 \perp} \cdot \mathbf{k}_{3 \perp}-k_{3}^{2} \mathbf{k}_{1 \perp} \cdot \mathbf{k}_{2 \perp}\right] v_{2,1}^{*} v_{3}
\end{aligned}
$$

and

$$
\begin{aligned}
& \left(\frac{\partial}{\partial t}+\mathbf{v}_{g 3} \cdot \nabla\right) v_{3} \simeq \frac{k_{z 3}^{2} c_{A}^{2}}{2 \omega_{3} k_{1 \perp} k_{2 \perp} k_{3 \perp} \omega_{c i}}\left[k_{1}^{2} \mathbf{k}_{3 \perp} \cdot \mathbf{k}_{2 \perp}\right. \\
& \left.\quad+k_{2}^{2} \mathbf{k}_{1 \perp} \cdot \mathbf{k}_{3 \perp}-k_{3}^{2} \mathbf{k}_{1 \perp} \cdot \mathbf{k}_{2 \perp}\right] v_{1} v_{2},
\end{aligned}
$$

where $v_{j}=\left|\mathbf{v}_{j}\right|$. Comparing the magnitude of the coupling coefficient in (15) and (16) with that of other MHD wave interactions when $k c_{A} \sim k c_{s} \sim \omega \ll \omega_{c i}$ (Brodin and Stenflo 1988), we note that the present coefficient is smaller by a factor of order $\omega / \omega_{c i}$. This is consistent with the previous result that the coupling coefficient for three shear Alfvén waves vanishes if the ideal MHD equations are applied. However, although the coefficient of the present process is small, we note that the interaction of three shear Alfvén waves may stay coherent for a much longer time, since the group velocities of the interacting waves are approximately equal. Thus, the present interaction process may be at least as important as other mechanisms in the regime $k c_{A} \sim k c_{s} \sim \omega \ll \omega_{c i}$.

\section{Summary and conclusion}

The Hall-MHD equations have previously successfully described the resonant nonlinear interactions between three magnetosonic waves, two magnetosonic and one Alfvén waves as well as one magnetosonic and two Alfven waves (Brodin and Stenflo 1988, 1990). Using the equations in Brodin and Stenflo (1990) one can in a comparatively simple way also consider the coupling between three Alfvén waves. It should however be stressed that using a rigorous description of Alfven waves, one finds dispersion relations (13) and (14) in Shukla and Stenflo (2000), which for some propagation directions differ significantly from the dispersion relation (7) derived from the Hall-MHD description in the present paper. The coupling coefficients here are also somewhat different from those of a rigorous derivation (Stenflo and Brodin 2006, Appendix A). However, the simplicity of the present analysis may have some pedagogical advantages as compared with other more complex attempts if the results are explored with due caution.

\section{References}

Bellan, P. M. 2012 J. Geophys. Res. 117, A12219.

Brodin, G. and Stenflo, L. 1988 J. Plasma Phys. 39, 277.

Brodin, G. and Stenflo, L. 1990 Contrib. Plasma Phys. 30, 413.

Brodin G., Stenflo, L. and Shukla, P. K. 2007 J. Plasma Phys.

73, 9 . 
Chmyrev, V. M., Bilichenko, S. V., Pokhotelov, O. A., Marchenko, V. A., Lazarev, V. I., Streltsov, A. V. and Stenflo, L. 1988 Phys. Scripta 38, 841.

Das, B. K., Kumar, S. and Sharma, R. P. 2012 Phys. Scripta 85, 035501.

Fedun, V. N., Yukhimuk, A. K. and Voitsekhovskaya, A. D. 2004 J. Plasma Phys. 70, 699.

Hirose, A., Ito, A., Mahajan, S. M., Ohsaki, S. 2004 Phys. Lett. A 330, 474.

Kumar, S. 2012 Astrophys. Space Sci. 337, 645.

Kumar, S. and Sharma, R. P. 2011 J. Plasma Phys. 77, 231.

Petviashvili, V. I. and Pokhotelov, O. A. 1992 Solitary Waves in Plasmas and in the Atmosphere. Berlin, Germany: Gordon and Breach.

Rudakov, L., Mithaiwala, M., Ganguli, G. and Crabtree C. 2011 Phys. Plasmas 18, 012307.

Ruderman, M. S. and Caillol, P. 2008 J. Plasma Phys. 74, 119.
Shukla, P. K. and Stenflo, L. 1995 Phys. Scripta T 60, 32.

Shukla, P. K. and Stenflo, L. 2000 J. Plasma Phys 64, 125.

Shukla, P. K., Rahman, H. U. and Sharma, R. P. 1982 J. Plasma Phys. 28, 125.

Stasiewicz, K., Bellan, P., Chaston, C., Kletzing, C., Lysak, R., Maggs, J., Pokhotelov, O., Seyler, C., Shukla, P., Stenflo, L., Streltsov, A. and Wahlund, J.-E. 2000 Space Sci. Rev. 92, 423.

Stenflo, L. and Brodin, G. 2006 J. Plasma Phys. 72, 143.

Sundkvist, D., Krasnoselskikh, V., Shukla, P. K., Vaivads, A., André, M., Buchert, S. and Réme, H. 2005 Nature 436, 825.

Voitenko, Yu. M. 1998 J. Plasma Phys. 60, 497.

Zhao, J. S., Wu, D. J. and Lu, J. Y. 2011 Phys. Plasmas 18, 032903.

Zhao, J. S., Wu, D. J., Yu, M. Y. and Lu, J. Y. 2012 Phys. Plasmas 19, 062901. 